BRIEF COMMUNICATION

\title{
Theoretical prediction of high melting temperature for a Mo-Ru-Ta-W HCP multiprincipal element alloy
}

\author{
Qi-Jun Hong $\mathbb{D}^{1 凶}$, Jan Schroers $\mathbb{D}^{2}$, Douglas Hofmann ${ }^{3}$, Stefano Curtarolo ${ }^{4}$, Mark Asta $\mathbb{i D}^{5,6}$ and Axel van de Walle (iD ${ }^{1}$
}

While rhenium is an ideal material for rapid thermal cycling applications under high temperatures, such as rocket engine nozzles, its high cost limits its widespread use and prompts an exploration of viable cost-effective substitutes. In prior work, we identified a promising pool of candidate substitute alloys consisting of $\mathrm{Mo}, \mathrm{Ru}, \mathrm{Ta}$, and $\mathrm{W}$. In this work we demonstrate, based on density functional theory melting temperature calculations, that one of the candidates, $\mathrm{Mo}_{0.292} \mathrm{Ru}_{0.555} \mathrm{Ta}_{0.031} \mathrm{~W}_{0.122}$, exhibits a high melting temperature (around $2626 \mathrm{~K}$ ), thus supporting its use in high-temperature applications.

npj Computational Materials (2021)7:1 ; https://doi.org/10.1038/s41524-020-00473-6

\section{INTRODUCTION}

Among refractory metals, rhenium demonstrates a unique combination of desirable thermodynamic and mechanical properties: a high melting point $(3448 \mathrm{~K})$, excellent high-temperature strength, a good ablation and creep resistance, and no ductile-tobrittle transition ${ }^{1,2}$. The concurrence of low-temperature ductility and high-temperature strength makes Re-based alloys competitive candidates for structural materials with applications such as rocket engine nozzles, which involve rapid thermal cycling under high temperatures. However, rhenium is a rare element and its high cost limits its widespread use ${ }^{3,4}$. This situation has prompted the exploration of practical and efficient replacement for rhenium in rocket engine nozzle applications.

Previously, we showed that a straightforward design principle that trades off average valence electron count and cost considerations is useful in recognizing a favorable group of possible substitutes, the Mo-Ru-Ta-W quaternary alloys ${ }^{5}$, based on a thermodynamic model that integrates electronic structure calculations with the Calphad framework ${ }^{6}$, as well as through experimental synthesis and structural characterization of samples chosen in a favorable composition range. The computational thermodynamic model enabled the identification of alloy compositions on a so-called Re-equivalent plane that also preserve rhenium's hcp structure, and thus its desirable mechanical properties ${ }^{7}$. In other words, based on a rigid-band model, promising alloys have a composition on a Re-equivalent plane on which the average number of valence electrons per atom equals that of Re. The accuracy of this simple picture was assessed through high-throughput ab initio thermodynamic calculations, and with comparisons to established binary phase diagrams sections, and further corroborated by experimental synthesis and structural characterization demonstrating single-phase multiprincipal-element hcp solid-solution samples selected from a promising composition range. This region includes the Mo-Ru-Ta-W quaternary alloys having an hcp structure at compositions near $\mathrm{Mo}_{0.3} \mathrm{Ru}_{0.512} \mathrm{Ta}_{0.064} \mathrm{~W}_{0.125}$ and near $\mathrm{Mo}_{0.292} \mathrm{Ru}_{0.555} \mathrm{Ta}_{0.031} \mathrm{~W}_{0.122}$, which straddle the Re-equivalent plane. Although $\mathrm{Ru}$ is also somewhat expensive, including it allows us to add more of the inexpensive elements such as Mo and $\mathrm{W}$, thus still resulting in a net cost reduction. These specific compositions were also synthesized experimentally and their mechanical properties investigated $^{5,8}$. We also showed that the proposed Ru-based alloys exhibit better thermal compatibility with Ir, which serves as an oxidation-resistant coating. ${ }^{\text {. }}$. However, it remains to be demonstrated that this proposed alloy exhibits a sufficiently high melting point for its intended application. An experimental determination of the melting point is complicated by the high temperatures involved. These alloys may have the potential to replace higher cost elements, like $\mathrm{Re}$, in relatively lowertemperature thruster applications where cost is a major driver but performance, such as ablation and erosion, is adequate.

In this work, we employ density functional theory (DFT) $)^{10-12}$ to calculate the melting temperature of $\mathrm{Mo}_{0.292} \mathrm{Ru}_{0.555} \mathrm{Ta}_{0.031} \mathrm{~W}_{0.122}$. For accuracy assessment and improvement, we also compute the melting points of its constituting elements. We use an efficient small-cell coexistence method ${ }^{13}$ and its implementation in the SLUSCHI code ${ }^{14}$, based on DFT molecular dynamics (MD), as shown in Fig. 1. This highly cost-effective and robust method allows us to perform expensive melting point calculations on multiple materials directly from DFT.

As shown in Table 1, our calculations suggest the need to use accurate pseudo-potentials where semicore elections $s$ and $p$ states were treated as valence states. With only valence elections, the DFT melting point errors were $210,170,330$, and $200 \mathrm{~K}$ for Mo, $\mathrm{Ru}, \mathrm{Ta}$, and $\mathrm{W}$, respectively. When semicore $p$ (including $s$ for $\mathrm{W}$ ) electrons were further relaxed, the errors were significantly reduced to $80,60,100$, and $170 \mathrm{~K}$, respectively. The high accuracy of the latter served as strong indication that our DFT melting point calculation model will also accurately generate melting properties of $\mathrm{Mo}_{0.292} \mathrm{Ru}_{0.555} \mathrm{Ta}_{0.031} \mathrm{~W}_{0.122}$, a promising Re-substitute alloy.

The hcp solid structure of $\mathrm{Mo}_{0.292} \mathrm{Ru}_{0.555} \mathrm{Ta}_{0.031} \mathrm{~W}_{0.122}$ was represented by a special quasi-random structure (SQS) ${ }^{15}$ of 64atom $\mathrm{Mo}_{19} \mathrm{Ru}_{35} \mathrm{Ta}_{2} \mathrm{~W}_{8}$. The mcsqs code ${ }^{16}$ was employed to generate the SQS. This SQS is then feed into the SLUSCHI code, which automates the melting point calculation process via the small-cell solid-liquid coexistence method. Duplicates of halfsolid-half-liquid coexistence simulations were carried out at various temperatures, and solid-liquid probability distribution

\footnotetext{
${ }^{1}$ School of Engineering, Brown University, Providence, RI 02912, USA. ${ }^{2}$ Department of Mechanical Engineering and Materials Science, Yale University, New Haven, CT 06511, USA. ${ }^{3}$ NASA Jet Propulsion Laboratory, California Institute of Technology, 4800 Oak Grove Dr., Pasadena, CA 91109, USA. ${ }^{4}$ Department of Mechanical Engineering and Materials Science and Center for Autonomous Materials Design, Duke University, Durham, NC 27708, USA. ${ }^{5}$ Department of Materials Science and Engineering, University of California, Berkeley, CA 94720, USA. ${ }^{6}$ Materials Sciences Division, Lawrence Berkeley National Laboratory, Berkeley, CA 94720, USA. ${ }^{\circledR}$ email: qhong@alumni.caltech.edu
} 

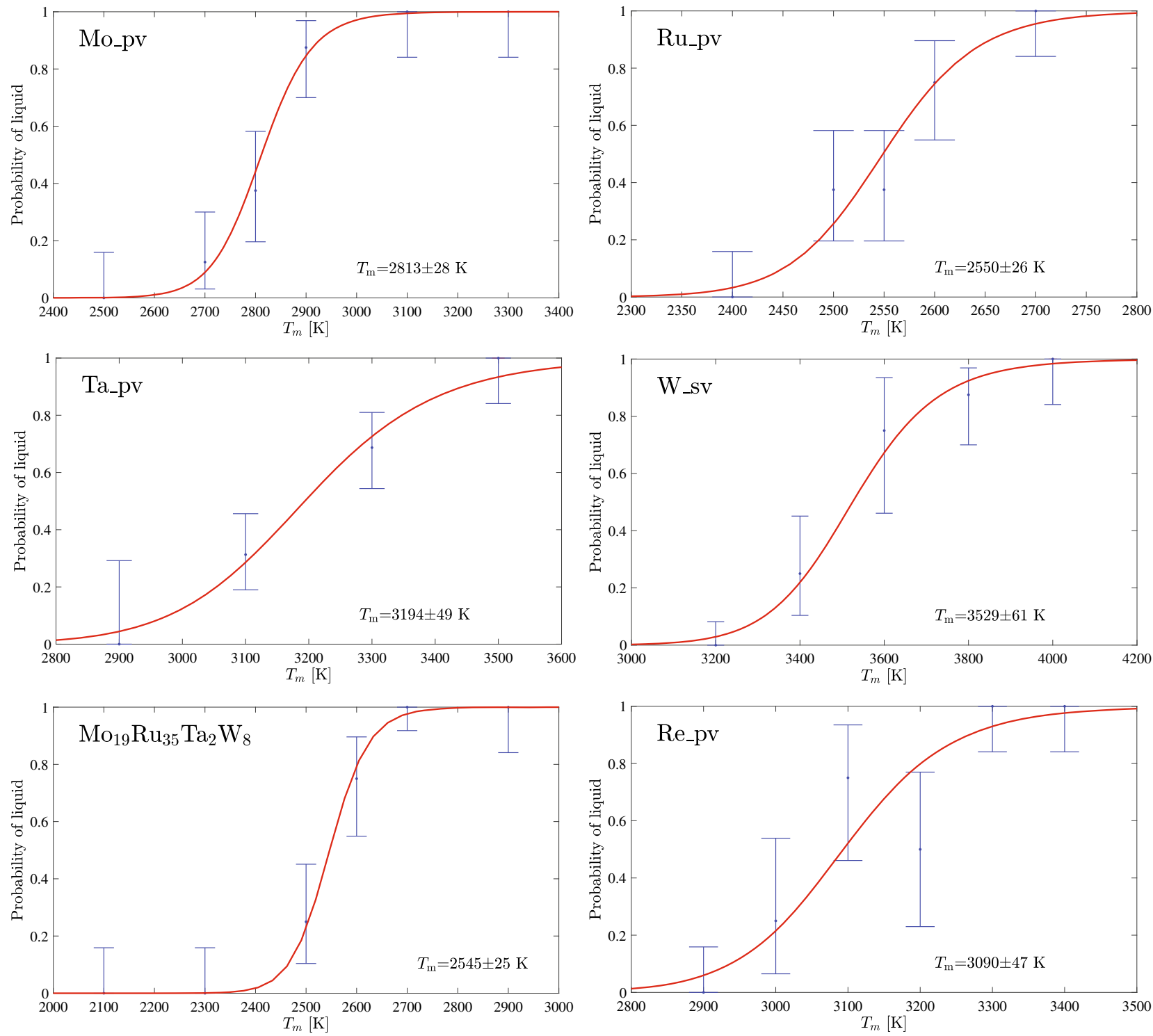

Fig. 1 Melting temperature calculations of $M o, R u, T a, W, R e$ and $\mathbf{M o}_{19} \mathbf{R u}_{35} \mathbf{T a}_{\mathbf{2}} \mathbf{W}_{\mathbf{8}}$. Duplicates of solid-liquid coexistence simulations were performed at various temperatures near melting. Distribution of trajectories that completely solidify or liquidify were then analyzed statistically (blue). Melting temperatures are formally inferred based on fitting (red) the statistical distributions. Error bars represent the standard error of binomial distribution.

was analyzed to rigorously infer the final melting temperature. The calculated meting point of $\mathrm{Mo}_{19} \mathrm{Ru}_{35} \mathrm{Ta}_{2} \mathrm{~W}_{8}$ is $2545 \pm 26 \mathrm{~K}$.

$A$ recent study suggests that, due to underbinding, GGA may systematically underestimate melting point and thus GGA value may serve as a lower boundary of melting point ${ }^{17}$. We here make a melting temperature correction on $\mathrm{Mo}_{0.292} \mathrm{Ru}_{0.555} \mathrm{Ta}_{0.031} \mathrm{~W}_{0.122}$, based on the known melting temperature errors of its alloying elements in pure form,

$$
\Delta T=\sum_{\mathrm{i}=\mathrm{Mo}, \mathrm{Ru}, \mathrm{Ta}, \mathrm{W}} \Delta T_{\mathrm{i}} x_{\mathrm{i}}
$$

where $x_{\mathrm{i}}$ is the composition of each alloying element, and $\Delta T_{\mathrm{i}}$ is the corresponding DFT melting point error. The correction is $81 \mathrm{~K}$, and the melting temperature of $\mathrm{Mo}_{0.292} \mathrm{Ru}_{0.555} \mathrm{Ta}_{0.031} \mathrm{~W}_{0.122}$ is thus estimated to be $2626 \mathrm{~K}$. This is (slightly) lower than the weighted average of the elemental melting points $(2850 \mathrm{~K})$, thus suggesting a eutectic topology.

While this melting temperature of $2626 \mathrm{~K}$ is significantly lower than that of rhenium $(3459 \mathrm{~K})$, this is not expected to be a limiting factor in applications where rhenium is used in conjunction with an iridium coating to improve oxidation resistance. Iridium's melting point is only $2719 \mathrm{~K}$ and thus becomes the more relevant limiting factor. Our proposed alloy's melting point would thus only lower the maximum operating temperature by about $100 \mathrm{~K}$. Note that a typical rhenium/iridium combustion chamber operates at temperatures from 1925 up to $2200{ }^{\circ} \mathrm{C}(2473 \mathrm{~K})^{18}$, a temperature range our alloy should still be able to withstand without melting.

We should point out that the small-size coexistence method is inherently capable of finding the correct solid crystal structure, because the solid-liquid interface facilitates the nucleation of the structure with the lowest free energy even if we initially assumed the wrong solid. In other words, the method itself serves as a corroboration to verify the correct solid structure. In our simulation, we confirm that $\mathrm{Mo}_{0.292} \mathrm{Ru}_{0.555} \mathrm{Ta}_{0.031} \mathrm{~W}_{0.122}$ remains in hcp structure, thus further corroborating our previous computational and experimental findings 5 .

In addition to Mo-Ru-Ta-W alloys, we also show that Ru-Re-Ta and Ru-Re-W alloys also have favorable high melting 
Table 1. Calculated melting temperatures, computational details and a comparison with experiment.

\begin{tabular}{|c|c|c|c|c|c|c|c|c|c|}
\hline Systems ${ }^{1}$ & \multicolumn{2}{|c|}{ Melting temperature } & \multicolumn{2}{|l|}{ Pseudopotentials $^{1}$} & $N^{3}$ & kmesh & $\Sigma n^{4}$ & $\begin{array}{l}\text { CPU } \\
\text { Hours }\end{array}$ & Days $^{5}$ \\
\hline Mo_pv, bcc & $2813 \pm 32$ & 2896 & Mo_pv 04Feb2005 & d801c707 & 128 & Baldereschi $^{6}$ & 36 & 29,000 & 16 \\
\hline Mo_v, bcc & $2684 \pm 45$ & 2896 & Mo 08Apr2002 & $1 \mathrm{e} 5 \mathrm{~d} 34 \mathrm{e} 8$ & 128 & Baldereschi & 22 & 8,000 & 8 \\
\hline Ru_pv, hcp & $2550 \pm 34$ & 2607 & Ru_pv 06Sep2000 & eda9ac22 & 152 & Baldereschi & 33 & 139,000 & 51 \\
\hline Ru_v, hcp & $2435 \pm 33$ & 2607 & Ru 06Sep2000 & 7379ca09 & 152 & Auto $20^{7}$ & 30 & 79,000 & 33 \\
\hline W_sv, bcc & $3529 \pm 64$ & 3695 & W_sv 04Sep2015 & $2 \mathrm{~d} 9 \mathrm{~d} 07 \mathrm{~b} 5$ & 128 & Baldereschi & 32 & 20,000 & 11 \\
\hline W_pv, bcc & $3469 \pm 45$ & 3695 & W_pv 06Sep2000 & $4 a 7 e 311 f$ & 128 & Baldereschi & 30 & 39,000 & 18 \\
\hline W_v, bcc & $3497 \pm 54$ & 3695 & W 08Apr2002 & b4472f00 & 128 & Auto 20 & 22 & 36,000 & 49 \\
\hline Re_pv, hcp & $3087 \pm 59$ & 3459 & Re_pv 06Sep2000 & 09822937 & 128 & Baldereschi & 25 & 150,000 & 43 \\
\hline Re_v, hcp & $3081 \pm 93$ & 3459 & Re 17Jan2003 & 86f87aff & 128 & Baldereschi & 21 & 59,000 & 30 \\
\hline $\mathrm{Ru}_{2} \mathrm{ReTa}, \mathrm{hcp}$ & $2164 \pm 57$ & - & M_v & & 152 & Auto 20 & 32 & 93,000 & 36 \\
\hline \multicolumn{10}{|c|}{$\begin{array}{l}1 \text { PAW and PBE were employed. "v", "pv", "sv" and dates denote different pseudo-potentials. "v" stands for valence elections only, while "pv" means semicore } \\
\text { elections } p \text { states were also treated as valence states. For W, even s semicore elections were relaxed. Supercell size was approximately } 20 \times 10 \times 10 \AA^{3} \text { in all } \\
\text { calculations. Default energy cutoffs were set and Pulay stress was included. } \\
2 \text { Title, date and type do not uniquely characterize a pseudo-potential. Here we identify a pseudo-potential with a } 32 \text {-bit CRC function of the POTCAR }{ }^{31} \text {. } \\
{ }^{3} \text { Number of atoms in the supercell. } \\
{ }^{4} \text { Total number of MD trajectories sampled. } \\
{ }^{5} \text { Physical time spent. } \\
6 \text { A special Baldereschi mean-value point in the Brillouin zone, (1/4,1/4,1/4). } \\
7 \text { Automatic generation of k-points (see VASP manual). }\end{array}$} \\
\hline
\end{tabular}

temperatures, as summarized in Table 1, while also remaining hcp structures. These results indicate that attempting to increase the alloy's melting point by replacing the element with the lowest melting point (Mo) by Re does appear to be an effective strategy.

It should be emphasized that the melting point reported here formally corresponds to the temperature, where the liquid and solid-free energies cross at a given fixed composition. This is the case because, over the timescale of our simulations, the species do not have the time to segrate from one phase to another, which can be readily verified by inspecting the final composition profile of the runs which terminate in a fully solid state. The real system may melt incongruently (with the solid and liquid having different compositions and the melting transformation taking place over a range of temperatures.) Our reported temperature lies in between the liquidus and the solidus, by construction.

In this system, the liquidus and solidus are likely very close to each other and thus close to our reported temperature. This is supported by the facts that (i) the alloy's main constituent (Ru) has a similar melting point $(2550 \mathrm{~K})$ as our composition of interest; (ii) additional calculations to estimate the melting at a composition near the middle point between $\mathrm{Mo}_{0.292} \mathrm{Ru}_{0.555} \mathrm{Ta}_{0.031} \mathrm{~W}_{0.122}$ (m.p. $2542 \mathrm{~K}$ ) and pure Ru suggest a melting point approximately $2500 \mathrm{~K}$. Therefore, the liquidus and solidus are indeed likely to both be very flat near the composition range of interest.

To summarize, we demonstrate, based on DFT calculations, that a potentially promising rhenium substitute alloy, $\mathrm{Mo}_{0.292} \mathrm{Ru}_{0.555^{-}}$ $\mathrm{Ta}_{0.031} \mathrm{~W}_{0.122}$, exhibits a very high melting temperature of $2626 \mathrm{~K}$, thus supporting its high-temperature applications. This prediction uses the known errors in the calculated melting temperature of the constituting elements to minimize any systematic bias. The calculations also corroborate our previous findings from both computation and experiment that $\mathrm{Mo}_{0.292} \mathrm{Ru}_{0.555} \mathrm{Ta}_{0.031} \mathrm{~W}_{0.122}$ has an hcp structure.

\section{METHODS}

Density functional theory melting point calculations

We use an efficient small-cell extension of the coexistence method ${ }^{13}$ and its implementation in the SLUSCHI code ${ }^{14}$, based on DFT MD. This highly efficient method makes it possible to perform, directly from first principles, expensive melting point calculations. The method runs solid-liquid coexisting simulations on small-size systems, and the melting temperatures are rigorously inferred based on statistical analysis of the system's fluctuations, namely, the temperaturedependence of the probability that simulations terminate with the system in a fully liquid state. This probability can be calculated analytically as a function of unknown thermodynamic parameters (including the melting point) that can then be determined from a fit to the observed temperature-dependent probabilities ${ }^{13}$. The accuracy (typically with an error smaller than $100 \mathrm{~K}$ ), robustness and efficiency of the method have been demonstrated in a range of materials ${ }^{13,14,19-24}$ In particular, the small-cell coexistence method ${ }^{13}$ and the SLUSCHI code ${ }^{14}$ was employed to computationally predict the material with the highest melting point, which was subsequently confirmed by independent experiments ${ }^{25-27}$. DFT calculations were performed by the Vienna Ab initio Simulation Package (VASP) ${ }^{28}$, with the projector-augmented-wave $(\mathrm{PAW})^{29}$ implementation and the generalized gradient approximation (GGA) for exchange-correlation energy, in the form known as Perdew, Burke, and Ernzerhof ${ }^{30}$. Since the simulations were performed at hightemperature conditions, we used accurate pseudo-potentials where the semicore $s$ and $p$ states were treated as valence states.

\section{DATA AVAILABILITY}

All data generated or analyzed during this study are included in this published article.

Received: 2 July 2020; Accepted: 28 November 2020; Published online: 04 January 2021 


\section{REFERENCES}

1. Campbell, I. E., Rosenbaum, D. \& Gonser, B. The availability, recovery and properties of rhenium metal. J. Less Common Met. 1, 185-191 (1959).

2. Carlen, J.-C. \& Bryskin, B. D. Rhenium-a qnique rare metal. Mater. Manuf. Process. 9, 1087-1104 (1994).

3. Fink, P. J., Miller, J. L. \& Konitzer, D. G. Rhenium reduction-alloy design using an economically strategic element. JOM 62, 55-57 (2010).

4. Wrona, A. et al. Properties of rhenium-based master alloys prepared by powder metallurgy techniques. Arch. Mater. Sci. Eng. 45, 95-101 (2010).

5. van de Walle, A., Sabisch, J. E., Minor, A. M. \& Asta, M. Identifying rhenium substitute candidate multiprincipal-element alloys from electronic structure and thermodynamic criteria. J. Mater. Res. 34, 3296-3304 (2019).

6. van de Walle, A., Sun, R., Hong, Q.-J. \& Kadkhodaei, S. Software tools for highthroughput CALPHAD from first-principles data. CALPHAD 58, 70-81 (2017).

7. de Jong, M. M. et al. Electronic origins of anomalous twinning in hexagonal close packed transition metals. Phys. Rev. Lett. 115, 065501 (2015).

8. Sabisch, J. E. C. Investigation of fundamental mechanical deformation mechanisms in rhenium for the development of replacement alloys. Doctoral dissertation, (University of California, Berkeley, 2017) https://digitalassets.lib.berkeley. edu/etd/ucb/text/Sabisch_berkeley_0028E_17612.pdf.

9. Sun, R., Asta, M. \& van de Walle, A. First-principles thermal compatibility between Ru-based Re-substitute alloys and Ir coatings. Comp. Mater. Sci. 170, 109199 (2019).

10. Hohenberg, P. \& Kohn, W. Inhomogeneous electron gas. Phys. Rev. B 136, B864 (1964).

11. Kohn, W. \& Sham, L. J. Self-consistent equations including exchange and correlation effects. Phys. Rev. 140, 1133 (1965).

12. Jones, R. O. \& Gunnarsson, O. The density functional formalism, its applications and prospects. Rev. Mod. Phys. 61, 689-746 (1989).

13. Hong, Q.-J. \& van de Walle, A. Solid-liquid coexistence in small systems: a statistical method to calculate melting temperatures. J. Chem. Phys. 139, 094114 (2013).

14. Hong, Q.-J. \& van de Walle, A. A user guide for SLUSCHI: solid and liquid in ultra small coexistence with hovering interfaces. CALPHAD 52, 88-97 (2016).

15. Zunger, A., Wei, S. H., Ferreira, L. G. \& Bernard, J. E. Special quasirandom structures. Phys. Rev. Lett. 65, 353-356 (1990).

16. van de Walle, A. et al. Efficient stochastic generation of special quasirandom structures. CALPHAD 42, 13-18 (2013).

17. Zhu, L.-F., Körmann, F., Ruban, A. V., Neugebauer, J. \& Grabowski, B. Performance of the standard exchange-correlation functionals in predicting melting properties fully from first principles: Application to al and magnetic ni. Phys. Rev. B 101, 144108 (2020).

18. Reed, B. D., Biaglow, J. A. \& Schneider, S. Iridium-coated rhenium radiation cooled rockets. NASA Technical Memorandum Number 107453 https://ntrs.nasa.gov/ citations/19970036365 (1997).

19. Hong, Q.-J., Ushakov, S., Navrotsky, A. \& van de Walle, A. Combined computational and experimental investigation of the refractory properties of $\mathrm{La}_{2} \mathrm{Zr}_{2} \mathrm{O}_{7}$. Acta Mater. 84, 275-282 (2015).

20. Hong, Q.-J. \& van de Walle, A. Prediction of the material with highest known melting point from ab initio molecular dynamics calculations. Phys. Rev. B 92, 020104 (2015).

21. Miljacic, L., Demers, S., Hong, Q.-J. \& van de Walle, A. Equation of state of solid, liquid and gaseous tantalum from first principles. CALPHAD 51, 133-143 (2015).

22. Hong, Q.-J. \& van de Walle, A. Reentrant melting of sodium, magnesium, and aluminum: general trend. Phys. Rev. B 100, 140102 (2019).

23. Guren, M. G. Ab Initio Molecular Dynamics Simulations of Melting Phase Relations in the System $\mathrm{CaO}-\mathrm{MgO}-\mathrm{SiO}_{2}$ at Pressures of the EarthÕs Lower Mantle MS Thesis (University of Oslo, 2017) https://www.duo.uio.no/handle/10852/58238.

24. Addington, C. K. Molecular Simulation of Phase Behavior, Interfacial Phenomena, and Pressure Effects in Porous Media. Doctoral dissertation (North Carolina State University, 2016) https://repository.lib.ncsu.edu/handle/1840.20/33235.

25. Cedillos-Barraza, O. et al. Investigating the highest melting temperature materials: a laser melting study of the TaC-HfC system. Sci. Rep. 6, 37962 (2016).
26. Buinevich, V. et al. Fabrication of ultra-high-temperature nonstoichiometric hafnium carbonitride via combustion synthesis and spark plasma sintering. Ceram. Int. 46, 16068 (2020).

27. Ushakov, S. V., Navrotsky, A., Hong, Q.-J. \& van de Walle, A. Carbides and nitrides of zirconium and hafnium. Materials 12, 2728 (2019).

28. Kresse, G. \& Furthmuller, J. Efficiency of ab-initio total energy calculations for metals and semiconductors using a plane-wave basis set. Comp. Mater. Sci. 6, 15-50 (1996).

29. Blöchl, P. E. Projector augmented-wave method. Phys. Rev. B 50, 17953-17979 (1994).

30. Perdew, J. P., Burke, K. \& Ernzerhof, M. Generalized gradient approximation made simple. Phys. Rev. Lett. 77, 3865-3868 (1996).

31. Friedrich, R. et al. Coordination corrected ab initio formation enthalpies. npj Comput. Mater. 5, 59 (2019).

\section{ACKNOWLEDGEMENTS}

This research was supported by National Science Foundation under grant DMR1835939, by Office of Naval Research under grants N00014-16-1-3124, N00014-17-12202, and N00014-20-1-2225, and by Brown University through the use of the facilities at its Center for Computation and Visualization. This work uses the Extreme Science and Engineering Discovery Environment (XSEDE), which is supported by National Science Foundation grant number ACI-1548562, via the resource Stampede2 at the Texas Advanced Computing Center (TACC) through allocation DMR050013N.

\section{AUTHOR CONTRIBUTIONS}

A.v.d.W. conceived the research. Q.-J. H. conducted the first-principles calculations and simulations. Q.-J. H. and A.v.d.W. wrote the manuscript with contributions from all authors. All authors have given approval to the final version of the manuscript.

\section{COMPETING INTERESTS}

The authors declare no competing interests.

\section{ADDITIONAL INFORMATION}

Correspondence and requests for materials should be addressed to Q.-J.H.

Reprints and permission information is available at http://www.nature.com/ reprints

Publisher's note Springer Nature remains neutral with regard to jurisdictional claims in published maps and institutional affiliations.

Open Access This article is licensed under a Creative Commons Attribution 4.0 International License, which permits use, sharing, adaptation, distribution and reproduction in any medium or format, as long as you give appropriate credit to the original author(s) and the source, provide a link to the Creative Commons license, and indicate if changes were made. The images or other third party material in this article are included in the article's Creative Commons license, unless indicated otherwise in a credit line to the material. If material is not included in the article's Creative Commons license and your intended use is not permitted by statutory regulation or exceeds the permitted use, you will need to obtain permission directly from the copyright holder. To view a copy of this license, visit http://creativecommons. org/licenses/by/4.0/.

(c) The Author(s) 2021 